\title{
Correction to: Deprescribing Opioids in Chronic Non-cancer Pain: Systematic Review of Randomised Trials
}

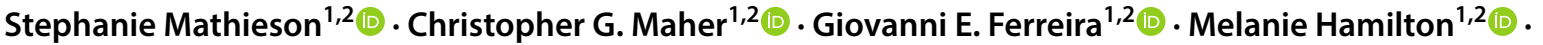

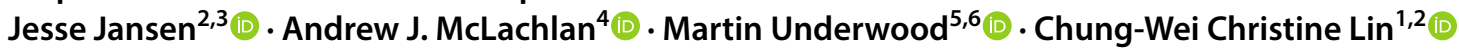

Published online: 29 September 2020

(c) Springer Nature Switzerland AG 2020

\section{Correction to: Drugs}

https://doi.org/10.1007/s40265-020-01368-y

Page 9, Fig. 2, which originally appeared as

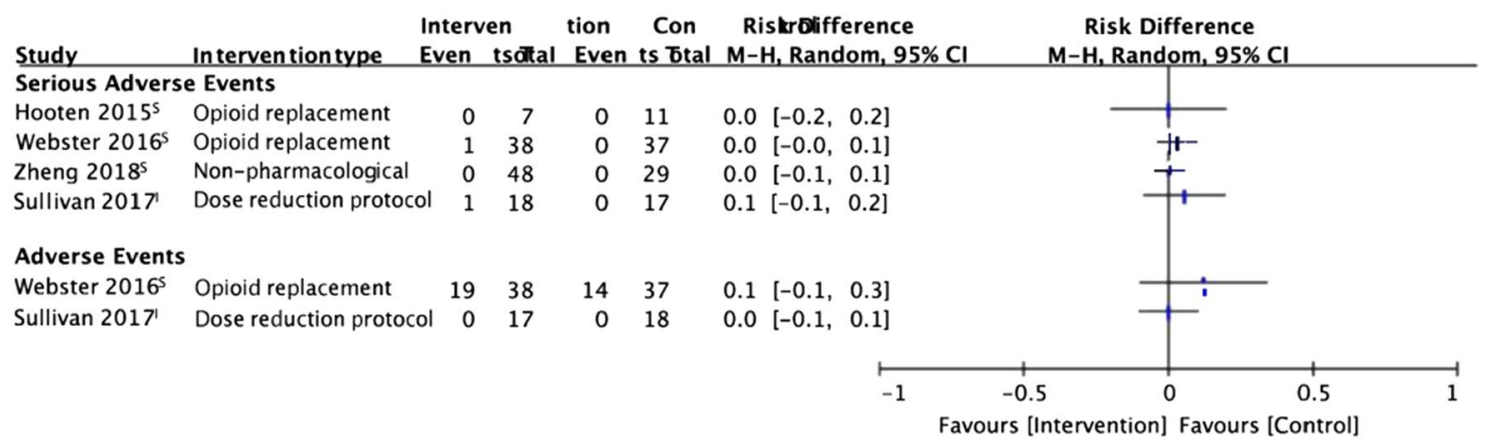

Footnote

$S=$ Short-term follow-up

$I=$ Intermediate-term follow-up

$L=$ Long-term follow-up

The original article can be found online at https://doi.org/10.1007/ s40265-020-01368-y.

Stephanie Mathieson

stephanie.mathieson@sydney.edu.au

Christopher G. Maher

christopher.maher@sydney.edu.au

Giovanni E. Ferreira

giovanni.ferreira@sydney.edu.au

Melanie Hamilton

melanie.hamilton@sydney.edu.au

Jesse Jansen

jesse.jansen@sydney.edu.au

Andrew J. McLachlan

andrew.mclachlan@sydney.edu.au

Martin Underwood

m.underwood@warwick.ac.uk

Chung-Wei Christine Lin

christine.lin@sydney.edu.au
1 Institute for Musculoskeletal Health, Level 10 North, King George V Building, Missenden Road, Camperdown, Sydney, Australia

2 Sydney School of Public Health, Faculty of Medicine and Health, The University of Sydney, Sydney, Australia

3 Sydney Health Literacy Lab, ASK-GP Centre for Research Excellence, Sydney, Australia

4 Sydney Pharmacy School, Faculty of Medicine and Health, The University of Sydney, Sydney, Australia

5 Warwick Clinical Trials Unit, University of Warwick, Coventry CV4 7AL, UK

6 University Hospitals Coventry and Warwickshire, Coventry CV2 2DX, UK 
Should appear as

\begin{tabular}{|c|c|c|c|c|c|c|c|c|c|c|c|}
\hline \multirow[b]{2}{*}{ Study } & \multirow[b]{2}{*}{ Intervention type } & \multicolumn{3}{|c|}{ Intervention } & \multicolumn{3}{|c|}{ Control } & \multirow{2}{*}{$\begin{array}{l}\text { Mean Difference } \\
\quad \text { IV, Random, } 95 \% \mathrm{CI}\end{array}$} & \multirow{2}{*}{\multicolumn{2}{|c|}{$\begin{array}{l}\text { Mean Difference } \\
\text { IV, Random, } 95 \% \mathrm{Cl}\end{array}$}} & \\
\hline & & Mean & SD & Total & Mean & & Total & & & & \\
\hline \multicolumn{12}{|c|}{ Dose reduction (morphine $\mathrm{mg} / \mathrm{day}$ ) from patient interventions } \\
\hline Kuri ta $2018^{\mathrm{s}}$ & Dose reduction protocol & -48.9 & 23.1 & 12 & -21.0 & 3.7 & 18 & $-27.9[-41.1,-14.7]$ & + & & \\
\hline Zheng $2018^{5}$ & Non-pharmacological & -30.2 & 62.3 & 36 & -52.3 & 112.3 & 27 & $22.1[-24.9,69.1]$ & & & \\
\hline Zheng $2018^{\prime}$ & Non-pharmacological & -47.4 & 62.2 & 25 & -63.3 & 111.9 & 20 & $15.9[-38.9,70.7]$ & & & \\
\hline Sullivan 2017 & Dose reduction protocol & -95.3 & 115.7 & 18 & -75.4 & 146.0 & 17 & $-19.9[-107.5,67.7]$ & & & \\
\hline \multicolumn{12}{|c|}{ Dose reduction (morphine $\mathrm{mg} /$ day) from patient interventions } \\
\hline \multirow[t]{2}{*}{ Liebschutz 201} & & -0.3 & 8.8 & 586 & 5.0 & 4.8 & 399 & $-5.3[-6.2,-4.5]$ & & & \\
\hline & & & & & & & & -200 & -100 & 100 & 200 \\
\hline Footnote & & & & & & & & Favou & Intervention & 1] Favours [C & \\
\hline
\end{tabular}

$\mathrm{S}=$ Short-term follow-up

Favours [Intervention] Favours [Control]

= Intermediate-term follow-up

$=$ Long-term follow-up

The original article has been updated. 